\title{
Whether atmospheric pressure changes predict variations in blood pressure
}

\begin{abstract}
Objective: The variations in blood pressure (BP) related to atmospheric pressure (AP) and the variables of age, gender and body mass index (BMI) in patients with essential hypertension (HTN) have not been studied in depth. This prospective 5-year study was conducted to assess the association between AP and blood pressure (BP) recorded in conditions of changing APs in the same room temperature.
\end{abstract}

Methods: BP was measured during routine follow-up visits of 182 hypertensive patients (102men and 80women; age range 65-92years) in an outpatient clinic with constant room temperature and APs that varied according to climate changes.

Results: The mean maximal systolic BP (SBP) difference $(\Delta)$ in AP between 1007 and 1019 millibars was $17.2 \pm 11.1$. The mean maximal diastolic $\mathrm{BP}(\mathrm{DBP})$ difference $(\Delta)$ in $\mathrm{AP}$ between 1007 and 1024millibars was $8.1 \pm 7.3 \mathrm{mmHg}$. BP and AP differences between 1007 and 1024 millibars correlated well with BMIs of 21-25 and 26-30 (They were $16 \pm 6.4 \mathrm{mmHg}$, $\mathrm{R}-0.42$ ). Pearson correlations were found in ages $65-69$ Rsbp -0.41 and 70-75 Rsbp-0.40, respectively $(\mathrm{p}<0.01)$ For DBP age subgroups-65-69years-Rdbp- $0.39(\mathrm{p}<0.01)$ and in subgroup70-76years R- 0.40( $<0.01)$.

Conclusion: The findings of this study demonstrated a direct relationship between AP above 1016 millibars and both SBP and DBP in HTN patients. Significant changes of the BP related to age (65-69years and 70-75years) and BMI (21-25 and 25-30) were found at AP differences between 1007 and 1024millibars.

Keywords: atmospheric pressure, seasonal variations, blood pressure, indoor blood pressure, barometric pressure
Volume 9 Issue 4 - 2017

\author{
Gideon Charach, Inbal Friedman, Hilla \\ Nochomovich, Ori Rogowski, Lior Charach, \\ Ronen Steinvil,Yoel Angel and Ori Argov \\ Department of Internal Medicine ?G?, Tel-Aviv Sourasky Medical \\ Center,Affiliated to the Sackler Faculty of Medicine, Tel Aviv \\ University, Tel-Aviv, Israel
}

Correspondence: Gideon Charach, Department of Internal
Medicine $\square C \square$, Tel-Aviv Sourasky Medical Center, 6 Weizman
Street, Tel-Aviv 6423906, Israel,Tel 972-3-6973768, Fax 972-36973775, Email drcharach@0। 2.net.il

Received: July 31, 2017 | Published: August 07, 2017
Abbreviations: AP, atmospheric pressure; HTN, hypertension; BP, blood pressure; BMI, body mass index; SBP, systolic blood pressure; DBP, diastolic blood pressure

\section{Introduction}

The influence of climate on arterial blood pressure (BP) has been demonstrated in several studies on hypertensive and normotensive patients of all ages. ${ }^{1-5}$ There is a tendency for both systolic (SBP) and diastolic blood pressure (DBP) to rise in the winter. ${ }^{1-6}$ Atmospheric pressure (AP) is the most objective weather factor because, regardless of being indoors or outdoors, it affects all objects in the same way.

The idea of an impact of climate on human health had been mentioned as early as the works of Hippocrates. ${ }^{7}$ In the Middle Ages, the "mighty air" was identified with the epidemics which occurred in Europe. Theories on climate changes and their impact on organisms originated in the period of Enlightenmentbut continuous recordings of weather conditions and their relationship with life parameters was not possible until the development of physics and technology, specifically, the invention of the thermometer and barometer, AP is the most objective meteorological factor that has the same influence on humans both indoors and outdoors. Several studies have confirmed that the flow of specific air masses is correlated with changes in wellbeing and the accompanying changes in $\mathrm{BP},{ }^{2-5,8-10}$ and that patients with chronic diseases are reportedly more liable to be affected by weather changes compared to healthy individuals. ${ }^{6}$ In patients with hypertension (HTN), even small fluctuations in BP influenced by weather can potentially result in serious neurological and cardiovascular complications. It has been suggested that a decrease in
AP increases BP via the sympathetic nervous system and the immune system. $^{\top}$

Previous report ${ }^{6}$ had shown greater seasonal BP fluctuations among elderly patients with HTN whose mean SBPs and DBPs were higher during the winter compared to the summer.

Their mean winter-summer difference was $31 \pm 19.1 \mathrm{mmHg}$ for SBP and $15 \pm 14.3$ for DBP $(\mathrm{p}<0.001) .{ }^{6}$ The specific cause of seasonal variation is not clear: one possibility that has been put forward is an inverse relationship between BP and the ambient temperature. ${ }^{1}$ According to some studies, more deaths occur in winter than in summer, with most of them being due to cardiovascular and cerebrovascular events among the elderly. ${ }^{6,11-13}$

The suggested cause of seasonal variation is an increased sympathetic nervous activity, as indicated by increased urinary and plasma catecholamines levels and increased sodium excretion. These have been implicated as potential mechanisms whereby BP increases in cold conditions. The extent of seasonal BP differences is, however, controversial. Stout et al., ${ }^{14}$ studied normotensive elderly in their own homes or in residential homes and reported that seasonal temperature differences were very low and statistically non-significant. Bruenen ${ }^{15}$ found significant differences only in seasonal systolic ambulatory daytime variations of BP in mildly hypertensive patients on treatment, but both the DBP ambulatory and office SBP and DBP seasonal differences were non-significant.

There is evidence that BP differs with various geographical areas and that it is lower in tropical climates compared to continental climates. ${ }^{12}$ The aim of this study was to investigate AP and related 
long-term variations in $\mathrm{BP}$ as well as its related complications in patients with HTN. BP measurements under subtropical East Mediterranean climatic conditions were related to age, body mass index (BMI) and gender.

\section{Methods}

\section{Patients}

A total of 230 outpatients with essential HTN were eligible for participation in the study. Inclusion criteria were elevated BP (SBP $130-200 \mathrm{mmHg}$ and DBP $90-115 \mathrm{mmHg}$ ) and age 40years or more. Exclusion criteria at all stages of the investigation included recent myocardial infarction, unstable angina, congestive heart failure, previous cerebrovascular accident, malignant disease, severe respiratory disease, renal failure (creatinine $>1.4 \mathrm{mg} / \mathrm{dl}$ ), anemia (hemoglobin $<12 \mathrm{~g} \%$ ), secondary HTN, hyper- or hypothyroid function and severe dementia.

All patients were put on a regimen of prescribed anti-HTN drugs: calcium channel blockers, diuretics, $\beta$-blockers, ACE inhibitors, ACE receptor blockers, and $\alpha$-blockers. They were advised to continue their usual daily consumption of caffeine, cigarettes or alcohol as well as fitness activities.

\section{Methods}

We reviewed the records of all the study patients who were followed due to their HTN in the hospital's outpatient clinic during a 5 -year period. The study was approved by the local ethical committee, and each of the 230 patients gave informed consent to participate in this prospective study.

The initial clinical visit included a physical examination, a fundus oculi evaluation, chest X-rays, blood tests (serum creatinine, electrolytes and complete blood count), and an electrocardiogram. On each examination day, after the subjects had been seated and had rested for 15 minutes, three consecutive readings were recorded by a mercury sphygmomanometer with a standard-sized cuff $(12 \mathrm{~cm}$ $X 35 \mathrm{~cm}$ ), and their mean values were calculated. Korotkoff phase 5 sounds were recorded as the level of diastolic pressure. Normal pressure was considered as SBP $\leq 140 \mathrm{mmHg}$ and DBP $\leq 90 \mathrm{mmHg}$, and all measurements were done in the same place and by the same examiner (G.C.) in order to minimize physiological and psychological influences. ${ }^{6,12-17}$ Follow-up visits were arranged at 3-month intervals during a 5-year period. If SBP measurements were $>180 \mathrm{mmHg}$ and DBP measurements were $>100 \mathrm{mmHg}$ diastolic, the patient received immediate treatment (sublingual captopril $25 \mathrm{mg}$ ). Spurious values of SBP $<80 \mathrm{mmHg}$ and DBP $<40 \mathrm{mmHg}$ and/or measurements of pulse pressure $<15 \mathrm{mmHg}$ were deleted. The patients were questioned about their general medical condition at each visit. They underwent a full medical examination every 6 months, similar to the one at the beginning of the study. The average indoor temperature was maintained by an air conditioner at $23^{\circ} \mathrm{C}$ during all four seasons. Outdoor environmental AP measurements were obtained from the national meteorological services (Beit Dagan).

\section{Statistical analysis}

The mean values of SBP and DBP for each participant and for and were recorded and calculated over the 5-year study period. The analysis included calculations of the Pearson correlations of the outcome and the variables of age and BMI, comparisons of mean values of the outcome variables in subgroups defined by gender, age and BMI, and multivariate analysis using a mixed linear model variant of the analysis of variants. The Bonferroni correction was used for estimating the $p$ values of differences between subgroups. All $p$ values were two-sided. A p value $<0.05$ was taken as being significant. All calculations were performed with SAS 8.12 (mainly Proc MIXED).

\section{Results}

Of the original 230 enrolled patients, 48 withdrew before the end of the first 3years of the study period for various causes (various diseases, death, poor compliance, change of residence, etc.) and their data were excluded from the final analysis. All of the remaining 182 patients were followed-up for at least 3years and up to 5years. The main demographic and clinical characteristics of the study group are shown in Table 1. The study population was comprised entirely of adults. Most of them were not obese, and there were no significant seasonal differences between their BMI values (results not shown).

Tables 2 and 3 show the AP and mean gender related absolute values of the SBP and DBP and their differences $(\Delta)$ under the same temperature conditions. The mean maximal SBP difference $(\Delta)$ in AP between 1007 and 1019 milligrams was $17.2 \pm 11.1 \mathrm{mmHg}$, $16.3 \mathrm{mmHg}$ for the males and $17.09 \mathrm{mmHg}$ for the females $(\mathrm{p}<0$. $001)$. The mean maximal DBP difference $(\Delta)$ in AP between 1007 and 1019 milligrams was $17.2 \pm 11.1 \mathrm{mmHg}, 8.2 \pm 11 \mathrm{mmHg}$ for the males and $7.02 \pm 3.1 \mathrm{mmHg}$ for the females. Most of the step-by-step AP elevations from 1007 to 1024 milligrams (1007 to 1009,1009 to 1013 , 1013 to 1016 , and 1019 to 1024) showed no significant differences in BP except for those between a $\triangle \mathrm{SBP}$ in an AP of 1016-1019 milligrams, which was $7.4 \pm 4.5 \mathrm{mmHg}$ and a $\triangle \mathrm{DBP}$ in an AP 10191024 milligrams which was $3.5 \pm 2.5 \mathrm{mmHg}(\mathrm{p}<0.01)$.

Table 4 describes the Pearson correlations in mean SBP and DBP variations between measurements in APs between 1007 and 1024millibars according to BMI. Significant differences were observed in the BMI values of $21-25$ and $26-30$ : they were $16 \pm 6.4 \mathrm{mmHg}$, $\mathrm{R}-0.42$ and $19 \pm 16.6 \mathrm{mmHg}, \mathrm{R}-0.4$, respectively $(\mathrm{p}<0.01)$ for $\mathrm{SBP}$ and $17 \pm 6.3 \mathrm{mmHg}, \mathrm{R}-0.38$ and $16 \pm 12.3 \mathrm{mmHg}, \mathrm{R}-41$, respectively, for DBP.

The Pearson correlations of mean SBP and DBP variations measurements in AP differences between 1007 and 1024millibar according to age are listed in Table 5. Good correlations with SBP were found for ages 65-69 years (Rsbp $-0.41, \mathrm{p}<0.01)$ and $70-75$ years (Rsbp-0.40, $\mathrm{p}<0.01$ ), and in DBP for ages 65-69years (-Rdbp -0.39, $\mathrm{p}<0.01)$ and $70-76$ years $(\mathrm{R}-0.40, \mathrm{p}<0.01)$.

\section{Discussion}

It has been reported in many studies that the impact of AP on organisms is not as prominent as the influence of season and temperature. ${ }^{1-6}$ The current study was performed under the same indoor conditions in terms of temperature, no wind and no direct sun exposure, although it was not possible to completely exclude the influence of season and temperature. ${ }^{17}$ The findings of the current study demonstrated a significant inverse relationship between AP and $\mathrm{BP}$ readings when AP values were higher than 1016millibars in a population of adults with HTN living in a subtropical Mediterranean climate.

Several studies have confirmed the inverse relationship between AP and arterial BP: the hypertensive patients whose measurements were taken during the days with higher APs had significantly higher $\mathrm{BP}$ values compared to those whose measurements were taken during the days with lower APs. ${ }^{17-19}$ Previous investigations showed that a low AP is also associated with an increase in the incidence of diseases of the cardiovascular system in which HTN constitutes a part of the 
pathogenesis. ${ }^{20-24} \mathrm{AP}$, particularly in combination with other weather conditions, influences the autonomic and endocrine systems. It has been commonly observed that a decrease in AP reduces blood oxygenation, and that reduced saturation leads to activation of the autonomic nervous and endocrine systems and, consequently, to an increase in BP. ${ }^{11,17}$ Among cerebrovascular events, a low AP is associated with an increase in the risk of hemorrhagic stroke and subarachnoid hemorrhage. ${ }^{6,20-23}$ It has been speculated that changes in AP may lead to changes in pressure within the vessel wall and facilitate its rupture, especially in an aneurysmatic modified vessel. ${ }^{23}$ Rupture of an aneurysm is facilitated by either an increase in BP inside the vessel or a rise of intramural pressure due to a decrease in intracranial pressure, and both situations might be associated with a decrease in AP.

Table I Main clinical and demographic characteristics of the study group

\begin{tabular}{lll}
\hline & Men $(\mathbf{n}=102)$ & Women $(\mathbf{n}=\mathbf{8 0})$ \\
\hline Age, years, mean \pm SD* & $75.2 \pm 16.2($ range 65-92) & $73.11 \pm 14.2($ range 65-9I) \\
Weight, kg mean $\pm S D^{*}$ & $82.4 \pm 11.2($ range 65-9I) & $68.6 \pm 8.3($ range 65-86) \\
Body mass index $\pm S D \dagger$ & $27.2 \pm 7.2($ range 19-43) & $23.1 \pm 12.4$ (range 18-37) \\
\hline
\end{tabular}

${ }_{p}<<0.001 ; \nmid p>0.05$

Table 2 Mean atmospheric pressure and gender-related (A) systolic blood pressure (SBP) and (B) diastolic blood pressure (DBP): absolute values and their differences $(\Delta)$ in the same room temperature conditions

\begin{tabular}{llll}
\hline SBP(A)Atmospheric pressure & SBP, mean \pm SD & BP mean \pm SD, & BP mean \pm SD, \\
\hline 1007 & & Males $\mathbf{n}=102$ & Females $\mathbf{n}=\mathbf{8 0}$ \\
1009 & $124 \pm 10.6^{*}$ & $126 \pm 11.3^{*}$ & $124 \pm 10.3$ \\
1013 & $126 \pm 13.6^{*}$ & $126 \pm 10.5^{*}$ & $122 \pm 9.8$ \\
1016 & $135 \pm 11.3^{*}$ & $136 \pm 9.3^{*}$ & $135 \pm 11.5^{*}$ \\
1019 & $134 \pm 17.3 \dagger$ & $135 \pm 14.6 \dagger$ & $135 \pm 13.8 \dagger$ \\
1024 & $141 \pm 23.6^{*}$ & $143 \pm 14.6^{*}$ & $141 \pm 12.5^{*}$ \\
\hline (B) DBP & $143 \pm 15.9^{*}$ & $140 \pm 16.2^{*}$ & $138 \pm 14.4^{*}$ \\
\hline & DBP, mean \pm SD & BP, mean \pm SD, & BP, mean $\pm \mathbf{S D}$, \\
\hline 1007 & & Males $\mathbf{n}=102$ & Females $\mathbf{n}=\mathbf{8 0}$ \\
1009 & $81.6 \pm 23.6$ & $83 \pm 22.1^{*}$ & $81 \pm 9.7$ \\
1013 & $83.0 \pm 15.7$ & $86 \pm 12.1^{*}$ & $83 \pm 17.1$ \\
1016 & $83.0 \pm 13.6^{*}$ & $86 \pm 26.1^{*}$ & $84 \pm 14.7^{*}$ \\
1019 & $84.1 \pm 8.5^{*}$ & $85 \pm 17.3^{*}$ & $83 \pm 8.5^{*}$ \\
1024 & $88 \pm 8.6^{*}$ & $88 \pm 9.5^{*}$ & $86 \pm 8.4^{*}$ \\
\hline
\end{tabular}

$*_{p}<0.00$ I; †p >0.05 SBP: Systolic Blood Pressure; DBP: Diastolic Blood Pressure

Table 3 Atmospheric pressure and gender-related SBP and DBP differences $(\Delta)$ in the same room temperature conditions-difference; SBP: Systolic Blood Pressure; DBP: Diastolic Blood Pressure; BMI: Body Mass Index *p<0.00I; $\nmid p>0.05$ (non-significant)

\begin{tabular}{llll}
\hline Atmospheric Pressure & Systolic BP, mean \pm SD & BP, mean \pm SD, & BP, mean \pm SD, \\
\hline$\Delta$ SBP 1007-1009 & & Males $\mathbf{n}=\mathbf{1 0 2}$ & Females $\mathbf{n = 8 0}$ \\
$\Delta$ SBP 1009-1013 & $2.1 \pm 0.3$ & $0.2 \pm 1.1$ & $1.0 \pm 0.3$ \\
$\Delta$ SBP I013-1016 & $1.2 \pm 0.4$ & $0.1 \pm 0.1$ & $1.2 \pm 0.2$ \\
$\Delta$ SBP 1016-1019 & $-1.2 \pm 1.3$ & $-1.3 \pm 1.5$ & $1.1 \pm 1.2$ \\
$\Delta$ SBP 1019-1024 & $7.4 \pm 4.5^{*}$ & $8.1 \pm 2.3^{*}$ & $6.2 \pm 1.1^{*}$ \\
$\Delta$ DBP 1007-1009 & $-3.2 \pm 1.8^{*}$ & $-3.2 \pm 1.3^{*}$ & $-3.4 \pm 0.8^{*}$ \\
$\Delta$ DBP 1009-1013 & $0.6 \pm 0.7$ & $1.0 \pm 0.4$ & $0.3 \pm 0.12$ \\
$\Delta$ DBP 1013-1016 & $0.5 \pm 0.07$ & $0.2 \pm 0.3$ & $1.0 \pm 0.3$ \\
$\Delta$ DBP 1016-1019 & $1.1 \pm 1.6^{*}$ & $1.2 \pm 1.8^{*}$ & $1.2 \pm 1.5^{*}$ \\
$\Delta$ DBP 1019-1024 & $3.5 \pm 4.5^{*}$ & $3.7 \pm 2.4^{*}$ & $1.2 \pm 1.1^{*}$ \\
\hline
\end{tabular}

Table 4 Pearson correlations of BMI and mean variations of SBP and DBP measurements in AP differences between 1007 and 1024 millibars according to BMI -difference; R: Pearson Correlation Index; SBP: Systolic Blood Pressure; DBP: Diastolic Blood Pressure; BMI: Body Mass Index *p<0.0I, †p>0.05 (non-significant)

\begin{tabular}{lllllll}
\hline BMI & $18-20$ & $2 I-25$ & $26-30$ & $31-35$ & $36-40$ & Mean \\
Pt. & $\mathrm{n}=37$ & $\mathrm{n}=46$ & $\mathrm{n}=53$ & $\mathrm{n}=28$ & $\mathrm{n}=18$ & $\mathrm{n}=182$ \\
$\Delta \mathrm{SBP} \pm \mathrm{SD}$ & $\mathrm{I} 4 \pm 8.2$ & $16 \pm 6.4$ & $19 \pm 16.6$ & $16 \pm 12.2$ & $15 \pm 14.4$ & $17 \pm 12.4$ \\
$\mathrm{R}$ & $-0.17 \dagger$ & $-0.42^{*}$ & $-0.40^{*}$ & $-0.16 \dagger$ & $-0.11 \dagger$ & $-0.17 \dagger$ \\
$\Delta \mathrm{DBP} \pm \mathrm{SD}$ & $7 \pm 4.3$ & $17 \pm 6.3$ & $16 \pm 12.3$ & $12 \pm 3.7$ & $9 \pm 5.6$ & $15 \pm 14.8$ \\
$\mathrm{R}$ & $-0.12 \dagger$ & $-0.38^{*}$ & $-0.4 I^{*}$ & $-0.17 \dagger$ & $-0.12 \dagger$ & $-0.19 \dagger$ \\
\hline
\end{tabular}


Table 5 Pearson correlations between age and mean variations of SBP and DBP measurements in AP differences between I007 and I024 millibars according to age -difference; R: Pearson Correlation Index; SBP: Systolic Blood Pressure; DBP: Diastolic Blood Pressure *p<0.0I, †p >0.05 (non-significant)

\begin{tabular}{llllllll}
\hline Age range, $y$ & $65-69$ & $70-75$ & $76-80$ & $8 I-85$ & $86-90$ & $>90$ & Mean \\
Patients $(\mathrm{n})$ & 43 & 48 & 38 & $3 \mathrm{I}$ & 16 & 6 & 182 \\
SBP & $13 \pm 12.3$ & $15 \pm 13.4$ & $8 \pm 4.5$ & $6 \pm 7.6$ & $6 \pm 5.3$ & $4 \pm 2.7$ & $10 \pm 7.4$ \\
R & $-0.4 I^{*}$ & $-040^{*}$ & $-0.11 \dagger$ & $-0.12 \dagger$ & $-0.09 \dagger$ & $-0.10 \dagger$ & -0.18 \\
DBP & $9 \pm 8.2$ & $7 \pm 1.6$ & $3 \pm 9.3$ & $4 \pm 2.2$ & $3 \pm 6.9$ & $3 \pm 3.7$ & $5 \pm 4.1$ \\
R & $-0.39 *$ & $-0.40^{*}$ & $-013 \dagger$ & $-0.13 \dagger$ & $-0.07 \dagger$ & $-0.08 \dagger$ & -0.19 \\
\hline
\end{tabular}

Various complications related to different climate conditions may lead to weather-related stress ${ }^{24,25}$ or even psychological stress, ultimately leading to an increase of BP-related strokes during the spring months. ${ }^{23,24,26-31}$

Changes of the BP that were related to age and BMI were clearly present among the hypertensive participants in the current study. The patients who were 65-69 and 70-75years of age showed significant BP changes compared to the other age groups ( $p>0.05)$ when APs were between 1007 and 1024. In addition, patients with a BMI 21-25 and 26-30 experienced significant changes of BP between AP changes of 1007-1024 compared to patients with a BMI of 18-29,30-35 and $36-40$ ( $p>0.05$ ). Many studies reported an increased risk of cardioand cerebrovascular events during the winter, without any relation to AP. ${ }^{26,28-33}$ The current results emphasize the impact of decreasing AP levels on the lowering of SBP and DBP.

The major limitation of this study is the relatively small sample of patients. Another limitation is the fact that only arterial pressure was taken into account in the statistical analysis, and any other ailments from which the patients may have been suffering were not considered. Studies on larger groups of patients are warranted to further clarify the issue of the influence of AP on blood pressure.

\section{Conclusion}

The results of this study confirmed a direct relationship between high ( $>1016$ millibars) AP and both SBP and DBP levels in hypertensive patients. Significant BP changes that were related to age (65-69years and 70-75years) and BMI (21-25 and 25-30) were found at AP differences 1007 and 1024millibar. It is recommended to maintain a closer and strict control of BP during periods of high AP.

\section{Acknowledgments}

None.

\section{Conflicts of interest}

Author declares there are no conflicts of interest.

\section{Funding}

None.

\section{References}

1. Brennan PJ, Greenberg G, Miall WE, et al. Seasonal variation in arterial blood pressure. Br Med J. 1982;285(6346):919-923.

2. Hozawa A, Kuriyama S, Shimazu T, et al. Seasonal variation in home blood pressure measurements and relation to outside temperature in Japan. Clin Exp Hypertens. 2011;33(3):153-158.

3. Saeki K, Obayashi K, Iwamoto J, et al. Stronger association of indoor temperature than outdoor temperature with blood pressure in colder months. J Hypertens. 2014; 32(8):1582-1589.

4. De Swiet M, Fayers PM, Shinebourn EA. Blood pressure in four and five year old children: the effects of environment and other factors in its measurement: the Brompton study. J Hypertens. 1984;2(5):501-505.
5. Jenner DA, English DR, Vandongen R, et al. Environmental temperature and blood pressure in 9-year-old Australian children. J Hypertens. 1987;5(6):683-686.

6. Charach G, Rabinovich P, Weintraub M. Seasonal changes in blood pressure and frequency of related complications in elderly Israeli patients with essential hypertension. Gerontology. 2004;50(5):315-321.

7. Hippocrate, Cornarius. On airs, waters, and places. Apud Johannem Elsevirium, Latin. 1658.

8. Yackerson NS, Bromberg L, Adler B, et al. Possible effects of changes in the meteorological state over semi-arid areas on the general well-being of weather-sensitive patients. Environ Health. 2012;11:26.

9. Morabito M, Crisci A, Orlandini S, et al. A synoptic approach to weather conditions discloses a relationship with ambulatory blood pressure in hypertensives. Am J Hypertens. 2008;21(7):748-752.

10. Ishikawa K, Niwa M, Tanaka T. Difference of intensity and disparity in impact of climate on several vascular diseases. Heart Vessels. 2012;27(1):1-9.

11. Curwen M. Excess winter mortality, temperature: a British phenomenon? Health Trends. 1990;22:169-175.

12. Feigin VL, Anderson CS, Anderson NE, et al. Is there a temporal pattern in occurrence of subarachnoid hemorrhage in the southern hemisphere? Pooled data from 3 large, population-based incidence studies in Australia, 1981-1997. Stroke. 2001;32:613-619.

13. Giaconi S, Ghione S, Palombo C, et al. Seasonal influences on blood pressure in high normal to mild hypertensive range. Hypertension. 1989;14(1):22-27.

14. Stout RW, Crawford V. Seasonal variations in fibrinogen concentrations among elderly people. Lancet. 1991;338(8758):338-313.

15. Brueren MM, Schouten BJ, Schouten HJ, et al. No relevant seasonal influences on office and ambulatory blood pressure. Data from a study in borderline hypertensive primary care patients. Am J Hypertens. 1998;11(5):602-605.

16. Abdulla K, Taka M. Climatic effects on blood pressure in normotensive and hypertensive subjects. Postgrad Med J. 1988;64(747):23-26.

17. Woodhouse PR, Khaw K, Plummer M. Seasonal variations of blood pressure and its ambient temperature in an elderly population. $J$ Hypertens. 1993;11(11):1267-1274.

18. Skrobowski A. The impact of selected atmospheric conditions on blood pressure. Wojskowa Akademia Medyczna, Polish, Warszawa, Poland. 1998.

19. Weinbacher M, Martina B, Bart T, et al. Blood pressure and atmospheric pressure. Ann N Y Acad Sci . 1996;783:335-336.

20. Charach G, Shochat M, Argov O, et al. Seasonal changes in blood pressure: cardiac and cerebrovascular morbidity and mortality. World $J$ Hypertens. 2013;3:1-8.

21. Danet S, Richard F, Montaye M, et al. Unhealthy effects of atmospheric temperature and pressure on the occurrence of myocardial infarction and coronary deaths. A 10-year survey: The LilleWorld Health Organization MONICA project (Monitoring trends and determinants in cardiovascular disease). Circulation. 1999;100(1):1-7. 
22. Dawson J, Weir C, Wright F, et al. Associations between meteorological variables and acute stroke hospital admissions in the west of Scotland. Acta Neurol Scand. 2008; 117(2):85-89.

23. Law HY, Wong GK, Chan DT, et al. Meteorological factors and aneurysmal subarachnoid haemorrhage in Hong Kong. Hong Kong Med J. 2009;15(2):85-89.

24. Turin TC, Kita Y, Murakami Y, et al. Higher stroke incidence in the spring season regardless of conventional risk factors: Takashima Stroke Registry, Japan, 1988-2001. Stroke. 2008;39(3):745-752.

25. Hessman-Kosaris A. The impact of weather on mood. Diogenes, Polish, Warszawa, Poland. 1998.

26. Kriszbacher I, Czopf L, Bódis J. The effects of seasonal variations and weather conditions on the occurrence of heart attacks in Hungary between 2000-2004. Orv Hetil . 2007;148(16):731-736.

27. Rumana N, Kita Y, Turin TC, et al. Seasonal pattern of incidence and case fatality of acute myocardial infarction in a Japanese population (from the Takashima AMI Registry, 1988 to 2003). Am J Cardiol. 2008;102(10):1307-1311.
28. Kristal-Boneh E, Harari G, Green MS. Seasonal change in 24-hour blood pressure and heart rate is greater among smokers than nonsmokers. Hypertension. 1997;30(3 Pt 1):436-441

29. Shinkawa A, Ueda K, Hasuo Y, et al. Seasonal variation in stroke incidence in Hisayama, Japan. Stroke. 1990;21(9):1262-1267.

30. Saeki K, Obayashi K, Iwamoto J, et al. Stronger association of indoor temperature than outdoor temperature with blood pressure in colder months. J Hypertens. 2014; 32(8):1582-1589.

31. Rusticucci M, Bettolli ML, de los Angeles M. Association between weather conditions and the number of patients at the emergency room in an Argentine hospital. Int J Biomet . 2002;46(1):42-51.

32. Seto TB, Mittleman MA, Davis RB, et al. Seasonal variation in coronary artery disease mortality in Hawaii: observational study. $B M J$. 1998;316(7149):1946-1947.

33. Lanska DJ, Hoffmann RG. Seasonal variation in stroke mortality rates. Neurology. 1999;52(5):984-990. 